\title{
ENERGY AND UTILITIES CONSUMPTION - AN APPRAISAL IN SUSTAINABLE BUILDINGS
}

\author{
B. B. Okon ${ }^{1}$ and V. E. Okon ${ }^{2, *}$

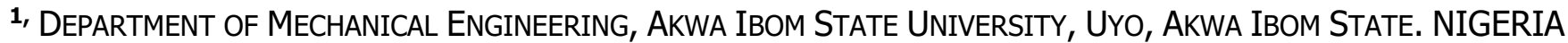 \\ 2, Dept. Of MeChanical Engineering, Michael OKPara Univ. Of AgRiculture, Umudike, ABia State, NIGERIA \\ E-mail addresses: ${ }^{1}$ engrbasseyokon@gmail.com, ${ }^{2}$ engrvik@gmail.com
}

\begin{abstract}
The rate of use of energy and utilities in cities is of great concern and necessitates appraisal especially when fossil fuel has been largely blamed for the global warming and climate change threats. This study investigates the involvement of professionals in the built environment during the design, operation and maintenance activities associated with energy and utilities consumption in buildings with a view to improving their management performance. Survey was directed to the building services engineers, estates and facilities managers within healthcare and educational sectors in the United Kingdom in pursuit of gathering energy and utilities consumption data. Issues that influence and support sustainable energy and utilities management practices were also sought during this study. A functional unit size of $100 \mathrm{~m}^{2}$ in each of the investigated buildings was considered as basis for analysis and comparison thus, the achievable results using sustainability indices option as an indicator for both healthcare and educational sectors are 0.74 and 0.64 . At the decommissioning stage of this buildings, the critical levels of service delivery are 0.27 and 0.24 respectively from both healthcare and educational sectors. These results are considerably satisfactory of the energy and utilities usage performance.
\end{abstract}

Keywords: Building services engineers, energy and utilities, maintenance, operation, sustainability

\section{INTRODUCTION}

Energy and utilities have consistently been the main source of livelihood for households, commercial and industrial use. For this reason, without the daily usage of energy and utilities, then there will be no meaningful economic activities as these resources are well established over the world as the mainstay for sustainable development success. Undebatable, the main function of energy and utilities (electricity, natural gas, fuel oil, and heating system) as well as water in this context is to provide sustainable, economic, reliable energy and utilities services for the desired comfort to households, commercial and industrial facilities users in support of the educational institution and healthcare activities [1]. The energy and utilities management within these sectors are design efforts which emphasised development policies and implementing initiatives that sustain optimal operation in the investigated educational institution and healthcare facilities.

It is an accepted fact that renewable energy and utilities including water conservation techniques and their maintenance management practices (MMP) are sustainable organisational measures which have attracted more attention in recent times [1-3]. Researchers have maintained that energy and utilities along with water conservation models option in these sectors consider all opportunities for achieving energy and water savings including replacing their facilities supply connections for effective service delivery [ 1 , 2]. In view of the above, the design and construction phases associated with buildings take-off influence a large extent of energy and other utilities use whereas, the operation and maintenance phases consume more energy utilities overtime than the later. Although, [1-3] noted that energy and water saving techniques options in this case are more desirable and

* Corresponding author, tel: +234- $902-742$ - 7974 
sustainable methods towards the development path of best practices in energy and utilities usage.

The extensive facilities (buildings) utilisation of energy management system requires more commitment to adapting local behaviours in order to decrease the consumption rate within the healthcare and educational sectors and this effort consciously intended to achieve sustainability agenda within the studied sectors. Sustainability, defined as meeting the needs of the present without compromising the ability of future generations to satisfying their own needs and has grown in significance and context across many business groups [4]. Increasingly, the healthcare and educational sectors are concerned with the impact emanated from energy and utilities usage and other activities on the environmental, social as well as economic sustainability [4 - 6].

The potential impact associated with energy and utilities consumption within the studied buildings and the management practices to achieving sustainability goals $[1,7]$ are well acknowledged. The significant influence of building services engineers, facilities and the estates management experts on energy and utilities consumption activities and the task to promote and implement the sustainability policies are also firmly established $[2,6-8]$. On the practical front, the building services engineers, estates and facilities managers in these sectors are responsible for the design, operation and maintenance of their organisation's vision and commitment towards the attainment of sustainability plan [6, 7]. In any case, the critical operational condition $(\mathrm{a} \mu)$ and the sustainability index (SI) level of facilities occur when the lifetime (t) yields $37 \%$ of the original (as-built facility) [12]. This obviously points to the fact that buildings and facilities usage should not exceed $37 \%$ of the original (as-built) status upon commissioning, operation and maintenance as well as decommissioning phases. This is because more resources at this point will be required to service these buildings without the return on investment. However, identifying the key issues capable of promoting the best and sustainable management practices also reveals the influence of these experts and their roles in energy and utilities consumption rate and operation within the investigated buildings in the United Kingdom.

\section{RESEARCH METHODS}

This study employed some mathematical computational methods and sustainability index model along with probability and the concept of set theory for sustainability analysis $[10,11]$. Also, a combination (C) and permutation ( $P$ ) functions method was applied to examine the energy and utilities consumption. Statistical analysis was also employed in the cause of this study according to [11].

\section{DATA COLLECTION}

An online self-administered survey through the UK's Freedom of Information (FOI) Act, (2000) was adopted for the data collection from both healthcare and educational buildings (facilities) in the UK. The overall aim was to gain more understanding of the building services and facilities management expert views regarding energy and utilities usage within the examined facilities. The survey was considered as the most appropriate method for objectively appraising the level of energy and utilities consumption activities and the expert opinions towards sustainability options in buildings management [9]. From 100 copies of administered questionnaires, 32 and 22 managers responded within the participating healthcare and educational sectors respectively. Within these facilities in both cases, an average of 200 users are considered. The energy and utilities category and the rate of consumption alongside the size of investigated buildings are considered in this analysis. Other information associated with the maintenance, operation and ancillary management practices in these buildings are also addressed in this paper. According to $[10,11]$ methods reported in Table 1 for data analysis were employed. All the employable analytical methods as applicable in this study are well documented.

\section{RESULTS AND DISCUSSION}

Findings from this investigation are presented in Table 1. The survey results in Figure 1, showed natural gas accounting for $26 \%$ of the energy and utilities consumption in the healthcare sector. This huge amount of gas used is probably because some of the participating healthcare facilities employ a combined heat and power (CHP) station option to generate electricity on site. As a consequence, a reasonable quantity of gas has been expended for electricity generation needed for services delivery.

Electricity consumption from this phase of study is 27 $\%$ of the overall utilities. This indicates the rate at which electricity is utilised within the hospitals. 
Table 1: Energy consumptions in the healthcare and educational sector.

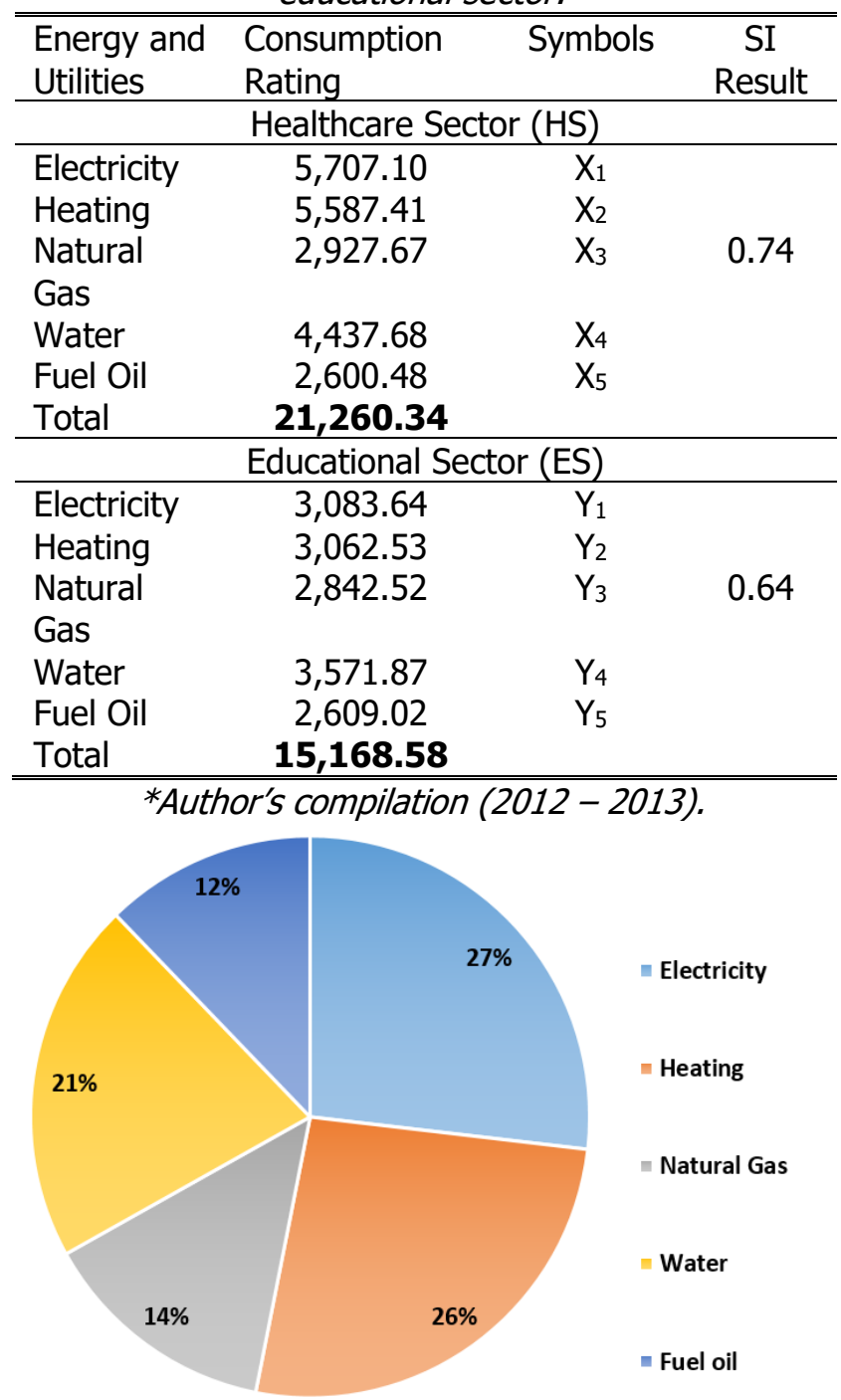

Figure 1: Consumption rating for health sector.

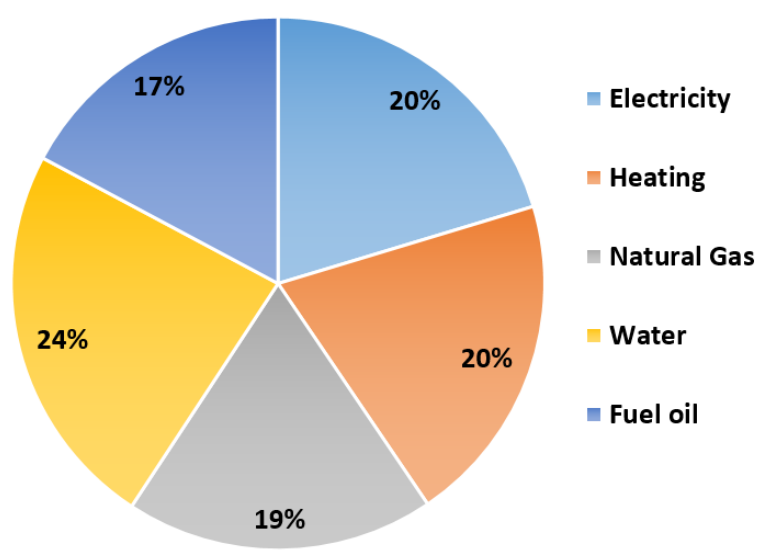

Figure 2: Consumption rating for educational sector

Water consumption in this study is $21 \%$ of the total utilities. This outcome explains the level at which water is utilised in the healthcare organisation. Heating and fuel oil energy within this sector account for about $26 \%$ of the entire investigated utilities. It should be noted that this outcome may not entirely represent a true picture of the energy and utilities consumption in each case but this is due to respondents' biases at the time of this investigation. Figure 2 shows that electricity and natural gas consumption from the educational sector is $20 \%$ of the total energy requirements. Similarly, water usage in the educational sector is $24 \%$ of the total energy and utilities, while heating and fuel oil contributes 36 $\%$ in this study. This finding represents a good spread of energy and utilities consumption statistics from the participating organisations in the survey. Obviously, these results are based on the data supplied by the respondents from the participating hospitals and schools during this study.

\section{CASE STUDY ANALYSIS}

A sustainability index model was applied to normalise the three themes of sustainable development: environmental, social and economic values associated with energy and utilities consumption. The application of sustainability index (SI) became necessary with a view to qualifying and quantifying values for accurate and reliable indices of sustainability within the healthcare and educational sectors. The sustainability index equation is given by equation (1) [10] as;

$$
\begin{aligned}
& {\left[n\left(E_{c v}\right) \cup n\left(E_{n v}\right) \cup n\left(S_{o v}\right)\right]=\left[n\left(E_{c v}\right)+n\left(E_{n v}\right)+n\left(S_{o v}\right)\right]} \\
& -\left[n\left(V_{v}\right)+n\left(B_{v}\right)+n\left(E_{q v}\right)+n\left(S_{u v}\right)\right]
\end{aligned}
$$

Where, $E_{\mathrm{cv}}-$ Economic values; $\mathrm{S}_{\mathrm{ov}}$ - Social values; $\mathrm{E}_{\mathrm{nv}}$ - Environmental values; $V_{v}$ - Viability values; $E_{q v}-$ Equitability values; $B_{v}-$ Bearability values; $S_{u v}-$ Sustainability values; $U-$ union and $n$ - number of values in set operation.

Equation (1) yields a sustainable engineering infrastructure (SEI) model which addresses and normalises sustainability to be within the limits of $0 \leq$ $\mathrm{S}_{\mathrm{uv}} \leq 1$ by applying the probability $(\mathrm{P})$ and the concepts of set theory into sustainability analysis [10]. Equation (1) also explains that energy and utilities consumption users at all times should consider the sustainability management option which revolves around the union and integration of the themes as expressed. By so doing, building services engineers and facilities management professionals should take the lead or be the drivers in the design, construction, operation and maintenance of facilities for optimum results in this context. Efforts in this direction will yield 
more fruitful results in view of sustainable development as the consciousness of experts are capable at promoting the ethics of sustainability agenda.

Analysing this study further, a combination (C) and permutation $(P)$ functions method in equation (2), [11] was applied to examine the energy and utilities consumption. Parameters and symbols were assigned to Table 1. For this reason, the expressions as contained in equations (2) and (7) were employed.

$$
\begin{aligned}
& N C n=\frac{N P m}{m !}=1 \\
& 5 C 3=\frac{5 P 3}{3 !}=1
\end{aligned}
$$

From the above expression in equations (2) and (3) $[10,11]$, where, $(N)$ denotes number of energy and utilities, $(m)$ expresses three top marked consumed energy and utilities. Then, $(n)$ represents the three highly consumed energy and utilities in both sectors. Sustainability index function satisfies the NCn number of energy and utilities indices derivations. Considerably, three top marked energy and utilities (electricity $X_{1}$, heating $X_{2}$ and water $X_{4}$ ) from the five investigated factors are presented in equation (3). Hence, for the healthcare sector:

$\left[X_{1}, X_{2}, X_{3}\right]\left[X_{1}, X_{3}, X_{4}\right]\left[X_{1}, X_{4}, X_{5}\right]\left[X_{2}, X_{3}, X_{4}\right]\left[X_{2}, X_{3}\right.$, $\left.X_{5}\right]\left[X_{3}, X_{4}, X_{5}\right]\left[X_{1}, X_{3}, X_{5}\right]\left[X_{1}, X_{2}, X_{5}\right]\left[X_{2}, X_{4}, X_{5}\right]\left[X_{1}\right.$ $\left.\mathrm{X}_{2}, \mathrm{X}_{4}\right]$

$$
\begin{aligned}
& \left(X_{1} \cup X_{2} \cup X_{3}\right)=\left[n\left(X_{1}\right)+n\left(X_{2}\right)+n\left(X_{3}\right)\right]-\left[n\left(X_{1} \cap X_{2}\right)\right. \\
& \left.+n\left(X_{1} \cap X_{3}\right)+n\left(X_{2} \cap X_{3}\right)+n\left(X_{1} \cap X_{2} \cap X_{3}\right)\right]
\end{aligned}
$$

In this study, five energy and utilities are examined from both healthcare and educational sectors as contained in Tables 1. The outcomes show three top marked energy and utilities from equation (3). These top marked energy and utilities were further analysed and this yields $\left[X_{1}, X_{2}, X_{4}\right]$. Taking the last function in equation (4), $\left[X_{1}, X_{2}, X_{4}\right]$ for the healthcare sector that is, electricity, heating and water as the highly consumed energy and utilities, the SI test applies grouping of energy and utilities consumption statistics from equation (6);

$$
\left(X_{1} \cup X_{2} \cup X_{4}\right)
$$

Equations (4), (5) and (6) explain the merits of sustainability index function option which quantifies the consumption rate index, equation (7). In this case, it is desirable for the building services engineers and facilities management experts to acquire this knowledge in pursuit of addressing energy and utilities resources management. Furthermore, this result simply indicates that the appraised facilities often consume electricity, heating and water more than other resources. This information was largely based on the supplied data.

$S I_{(H S)}=\left(\begin{array}{llllll}5,707.10 & \cup & 5,587.41 & \cup & 4,437.68\end{array}\right)=$ $15,732.19$

In the education sector also, considering the parameters $\left(Y_{1}, Y_{2}, Y_{4}\right)$, that is, electricity, heating and water as the main energy and utilities yield;

$3083.64 \cup 3062.53 \cup 3571.87$

$S I_{(E S)}=(3083.64 \cup 3062.53 \cup 3571.87)=9,718.04$

In this analysis, equations (7) and (8) have presented the energy and utilities SI results for the healthcare and educational sectors. Further explanation on the studied buildings show that energy and utilities are more consumed in the healthcare sector than the educational sector. This obviously is justifiable because the healthcare sector runs all through the year without downtime but the educational sector often operates on term vacations. On this note, the achievable results are reasonable.

\section{SUSTAINABILITY PERFORMANCE ANALYSIS - STUDIED BUILDINGS}

A sustainability performance study was conducted within the investigated facilities to ascertain their current level of operation and the critical level of services delivery. In this context, it explains the operational phase of a facility when the services delivery is no longer achieved as predicted. This situation thereby suggests decommissioning [1, 2]. More explanation could be at this critical level in facilities operations that, substantial amount of utilities (energy, heating and water) are consumed and huge financial expenses are anticipated to address the refurbishment challenge with a thorough corrective maintenance culture option $[1,2,8]$. The critical operational condition (a $\mu$ ) and the SI level of the investigated facilities occur when the lifetime ( $t$ ) as presented in equation [9] yields $37 \%$ of the original (as-built facility), considering the fact that SI function option was earlier used for the quantification of the assessed energy and utilities resources as shown in equation (10).

$$
\begin{gathered}
\mathrm{t}=\mathrm{a} \mu \\
\operatorname{SI}_{c}(\mathrm{t})=\operatorname{SIo}\left(\ell^{-1}\right)
\end{gathered}
$$


Where; (c) and (o) represent the construction and operational phases, and $(\mathrm{t})$ signifies the lifetime of the investigated facilities in both healthcare and educational sectors. Equation (10) was employed to test whether energy and utilities consumption within the studied facilities were economical viable or not. Considering the fact that sustainable facilities are not likely to consume huge amount of energy and utilities resources during the operational phase. Recalling the SI results in Table 1, and integrating them into equation (10), the healthcare and educational sectors yield 0.27 and 0.24 respectively as their critical point of services delivery in these facilities.

\section{CONCLUSION}

In this paper, the SI results in energy and utilities use from the healthcare and educational sectors are correspondingly 0.74 and 0.64 . The facilities performance analyses at the critical level of services delivery, that is, when these facilities are due decommissioning are 0.27 and 0.24 through the applied method [11]. Obviously, these results are based on the data supplied by the respondents from the participating hospitals and schools during this investigation. It could further be deduced that energy and utilities consumption within the investigated facilities are quite enormous and routine appraisal becomes desirable. This study, in addition addresses not only energy and utilities resources usage but also the entire facilities performance, that is, from cradleto-grave (design, construction, operation/maintenance and the decommissioning) stages in order to render professional expertise as appropriate in the direction of achieving resources gains. Pursuant to this, the concepts of engineering economics, maintenance management culture and sustainability ethics in managing infrastructure systems are successfully applied in this study.

\section{ACKNOWLEDGMENT}

This study was supported by the healthcare and educational sectors in the United Kingdom and the assistance of buildings services and facilities managers within the healthcare and educational sectors are appropriately acknowledged in this paper.
Also, the Akwa Ibom State University of Technology (AKSU) in Nigeria is lauded for their support.

\section{REFERENCES}

[1]. Cheryl-Gomez, P. E. (2008). 'Energy and Utilities', University of Virginia, Facilities Management, Annual Reports, United States of America.

[2]. Shah, S. (2007). Sustainable Practice for the Facilities Manager'. Blackwell Publishing, Oxford, England, United Kingdom.

[3]. Abdeen, M. O. (2011). 'Energy and environment: applications and sustainable development', British Journal of Environment \& Climate Change, 1, 3, pp.118-158, England.

[4]. 'World Commission on Environment and Development', (1987). Brutland Report.

[5]. Lindsey, T. C.' (2011). Sustainable principles: common values for achieving sustainability'. Journal of Cleaner Production, 19, 5, pp.561-565.

[6]. Elmualim, A., Vale, R and Kwawu, W. (2012). 'Discerning policy and drivers for sustainable facilities management practice'. International Journal of Sustainable Built Environment, 1, pp.16 $-25$.

[7]. Wood, B. (2006) 'The role of existing buildings in the sustainability agenda'. Facilities $24(1 / 2)$, pp. 61- 67.

[8]. Cheshire, D and Maunsell, F. (2007). 'Chartered Institution of Building Services Engineers'. Sustainability meets ASHRAE Guide.

[9]. KPMG, (2008). International Survey of Corporate Responsibility Reporting' Amstelveen.

[10]. Okon, B. B., Ekpo, S., and Elhag, T. S. M (2010). 'A Sustainable Engineering Infrastructure Model for the 21st Century', WCE. London, United Kingdom

[11]. Stroud, K. A. (2010). Engineering Mathematics'. Fifth Edition, Industrial Press Incorporated, New York, United States of America.

[12]. Okon, B. B. \& Okon, V. E. (2017): Building Services Equipment and Effective Maintenance Culture: The Experts' Standpoint. Journal of Mechanical Engineering and Automation, IJME20171122-2, United States of America. 